\title{
A note on the complete convergence for sequences of pairwise NQD random variables
}

\author{
Haiwu Huang ${ }^{1,2^{*}}$, Dingcheng Wang ${ }^{1}$, Qunying $W^{2}$ and Qingxia Zhang ${ }^{1}$
}

\author{
* Correspondence: \\ huanghaiwu@glite.edu.cn \\ ${ }^{1}$ School of Mathematics Science, \\ University of Electronic Science \\ and Technology of China, \\ Chengdu 610054, PR China \\ Full list of author information is \\ available at the end of the article
}

\begin{abstract}
In this paper, complete convergence and strong law of large numbers for sequences of pairwise negatively quadrant dependent (NQD) random variables with nonidentically distributed are investigated. The results obtained generalize and extend the relevant result of Wu (Acta. Math. Sinica. 45(3), 617-624, 2002) for sequences of pairwise NQD random variables with identically distributed.
\end{abstract}

2000 MSC: 60F15.

Keywords: pairwise NQD random variable sequences, complete convergence, almost sure convergence

\section{Introduction}

In many stochastic models, the assumption of independence among random variables is not plausible. So, it is necessary to extend the concept of independence to dependence cases, one of these dependence structures is negatively quadrant dependent (NQD) random variables.

The concept of NQD random variables was introduced by Lehmann [1].

Definition 1.1 [1] Two random variables $X$ and $Y$ are said to be NQD random variables if for any $x, y \in R$,

$$
P(X<x, Y<y) \leq P(X<x) P(Y<y) .
$$

A sequence of random variables $\left\{X_{n} ; n \geq 1\right\}$ is said to be pairwise NQD random variables if for all $i \neq j, i, j \in N, X_{i}$ and $X_{j}$ are NQD random variables.

Obviously, a sequence of pairwise NQD random variables is a family of very wide scope, which contains sequences of pairwise independent random variables. Many known types of negative dependence such as negatively orthant dependent (NOD) random variables and negatively associated (NA) [2] random variables are the most important and special cases of pairwise NQD random variables. So, it is very significant to study probabilistic properties of this wider pairwise NQD class. Since the concept of NQD random variables was introduced by Lehmann, many researchers have been established a large number of limit results for pairwise NQD random variable sequences. We can refer to Matula [3] for the Kolmogorov strong law of large numbers, Wang et al. [4] for the Marcinkiewicz strong law of large numbers and Baum and Katz complete convergence theorem, Wu [5] for Three series theorem, the complete convergence theorem and Marcinkiewicz strong law of large numbers, Chen [6] for the

(c) 2011 Huang et al; licensee Springer. This is an Open Access article distributed under the terms of the Creative Commons Attribution License (http://creativecommons.org/licenses/by/2.0), which permits unrestricted use, distribution, and reproduction in any medium, provided the original work is properly cited. 
Kolmogorov-Chung-type strong law of large numbers, Gan and Chen [7] for the strong stability of Jamison's weighted sums. But most of their results were achieved under the identically distributed condition and some results were obtained even under the condition of $\phi^{*}(1)<1$, where

$$
\varphi^{*}(1)=\lim _{m \rightarrow \infty} \sup _{n \geq m} \sup _{A \in F_{m}^{n}, B \in F_{n+1}^{\infty}, P(A)>0}|P(B \mid A)-P(B)| .
$$

When these are compared with the corresponding results of independent random variable sequences, there still remains much to be desired.

The concept of complete convergence of a sequence of random variables was introduced by Hsu and Robbins [8]. A sequence of random variables converges completely to the constant $c$ if

$$
\sum_{n=1}^{\infty} P\left(\left|X_{n}-c\right|>\varepsilon\right)<\infty \quad \text { for any } \quad \varepsilon>0 .
$$

In view of the Borel-Cantelli lemma, this implies that $X_{n} \rightarrow c$ almost surely. Hence, the complete convergence is a very important tool in establishing almost sure convergence of summation of random variables. Hsu and Robbins [8] proved that the sequence of arithmetic means of independent and identically distributed random variables converges completely to the expected value if the variance of the summands is finite. Erdös [9] proved the converse. The result of Hsu-Robbins-Erdös is a fundamental theorem in probability theory and has been intensively investigated in several directions by many authors in the past decades. One of the most important results is Baum and Katz [10] strong law of large numbers.

Theorem A [10] Let $\alpha p \geq 1, p>2$, and let $\left\{X_{n} ; n \geq 1\right\}$ be a sequence of independent and identically distributed random variables and $E\left|X_{1}\right|^{p}<\infty$. If $\frac{1}{2}<\alpha \leq 1$, assume that $E X_{n}=0, n \geq 1$. Then

$$
\sum_{n=1}^{\infty} n^{\alpha p-2} P\left(\max _{1 \leq j \leq n}\left|\sum_{i=1}^{j} X_{i}\right|>\varepsilon n^{\alpha}\right)<\infty \quad \text { for all } \varepsilon>0 .
$$

$\mathrm{Wu}$ [5] extended the result of Baum and Katz [10] to pairwise NQD random variable sequences with identically distributed under the condition of $\alpha p>1$ and $0<p<2$.

Theorem B [5] Let $\alpha p>1,0<p<2$, and let $\left\{X_{n} ; n \geq 1\right\}$ be a sequence of identically distributed pairwise NQD random variables and $E\left|X_{1}\right|^{p}<\infty$. If $\frac{1}{2}<\alpha \leq 1$, assume that $E X_{n}=0, n \geq 1$. Then

$$
\sum_{n=1}^{\infty} n^{\alpha p-2} P\left(\max _{1 \leq j \leq n}\left|\sum_{i=1}^{j} X_{i}\right|>\varepsilon n^{\alpha}\right)<\infty \text { for all } \varepsilon>0 .
$$

In this article, complete convergence and strong law of large numbers for sequences of pairwise NQD random variables with non-identically distributed are investigated. The main results obtained generalize and extend the relevant result of Wu [5] for sequences of pairwise NQD random variables with identically distributed. 


\section{Main results}

Throughout this article, the symbol $c$ denotes a positive constant which is not necessarily the same one in each appearance, $a_{n}=O\left(b_{n}\right)$ will mean $a_{n} \leq c\left(b_{n}\right)$, and $a_{n} \ll b_{n}$ will mean $a_{n}=O\left(b_{n}\right)$.

We will use the following concept in this article. Let $\left\{X_{n} ; n \geq 1\right\}$ be a sequence of NQD random variables and let $X$ be a nonnegative random variable. If there exists a constant such that

$$
\sup _{n \geq 1} P\left(\left|X_{n}\right| \geq t\right) \leq c P(X \geq t) \text { for all } t \geq 0 .
$$

Then, $\left\{X_{n} ; n \geq 1\right\}$ is said to be stochastically dominated by $X$ (briefly $\left\{X_{n} ; n \geq 1\right\} \pi X$ ). Clearly if $\left\{X_{n} ; n \geq 1\right\} \pi X$, then for $0<p<\infty, E\left|X_{n}\right|^{p} \leq c E X^{p}$ for any $n \geq 1$. Now we state the main results of this article.

Theorem 2.1 Let $\left\{X_{n} ; n \geq 1\right\}$ be a sequence of non-identically distributed pairwise NQD random variables with $\left\{X_{n} ; n \geq 1\right\} \pi X$ and $E|X|^{p}<\infty, 0<p<2$ and let $S_{n}=\sum_{i=1}^{n} X_{i}$. When $1 \leq p<2$, assumes that $E X_{n}=0, n \geq 1$. Then

$$
\sum_{n=1}^{\infty} n^{-1} P\left(\max _{1 \leq j \leq n}\left|S_{j}\right|>\varepsilon n^{1 / p} \log n\right)<\infty \text { for all } \varepsilon>0 .
$$

Theorem 2.2 Let $\left\{X_{n} ; n \geq 1\right\}$ be a sequence of non-identically distributed pairwise NQD random variables with $\left\{X_{n} ; n \geq 1\right\} \pi X$ and $E|X|^{p}<\infty, 0<p<2$ and let $S_{n}=\sum_{i=1}^{n} X$. When $1 \leq p<2$, assumes that $E X_{n}=0, n \geq 1$. Then

$$
\lim _{n \rightarrow \infty} \frac{S_{n}}{n^{1 / p} \log n}=0 \quad \text { a.s. }
$$

\section{Proof of main results}

To prove our main results, we need the following lemmas.

Lemma 3.1 [1] Let $X$ and $Y$ be NQD, then

(1) $E X Y \leq E X E Y$;

(2) $P(X>x, Y>y) \leq P(X>x) P(Y>y)$, for any $x, y \in R$;

(3) If $f, g$ are both nondecreasing (or non-increasing) functions, then $f(X)$ and $g(Y)$ are NQD.

Lemma 3.2 [5] Let $\left\{X_{n} ; n \geq 1\right\}$ be a sequence of pairwise NQD random variables with $E X_{n}=0$ and $E X_{n}^{2}<\infty$ for all $n \geq 1$. Then

(1) $E\left(\sum_{i=1}^{n} X_{i}\right)^{2} \leq \sum_{i=1}^{n} E X_{i}^{2}$, for all $n \geq 1$;

(2) $E \max _{1 \leq j \leq n}\left(\sum_{i=1}^{j} X_{i}\right)^{2} \leq \frac{4 \log ^{2} n}{\log ^{2} 2} \sum_{i=1}^{n} E X_{i}^{2}$, for all $n \geq 1$;

Proof of Theorem 2.1 Let $X_{i}^{(n)}=X_{i} I\left(\left|X_{i}\right| \leq n^{1 / p}\right)+n^{1 / p} I\left(X_{i}>n^{1 / p}\right)-n^{1 / p} I\left(X_{i}<-n^{1 / p}\right)$, $S_{j}^{(n)}=\sum_{i=1}^{j} X_{i}^{(n)}$, for any $i \geq 1$. For all $\varepsilon>0$, first we show that

$$
n^{-1 / p} \max _{1 \leq j \leq n}\left|\sum_{i=1}^{j} E X_{i}^{(n)}\right| \rightarrow 0 .
$$


(1) when $0<p<1$, then

$$
\begin{aligned}
n^{-1 / p} \max _{1 \leq j \leq n}\left|\sum_{i=1}^{j} E X_{i}^{(n)}\right| & \leq n^{-1 / p} \sum_{i=1}^{n}\left|E X_{i}^{(n)}\right| \\
& =n^{-1 / p} \sum_{i=1}^{n}\left|E X_{i} I\left(\left|X_{i}\right| \leq n^{1 / p}\right)\right|+\sum_{i=1}^{n} P\left(\left|X_{i}\right|>n^{1 / p}\right) \\
& \ll n^{1-1 / p} E|X| I\left(|X| \leq n^{1 / p}\right)+2 n P\left(|X|>n^{1 / p}\right) \\
& \leq n^{1-1 / p} E|X| I\left(|X| \leq n^{1 / p}\right)+2 n \frac{E|X|}{n^{1 / p}} \\
& \leq n^{1-1 / p} E|X| I\left(|X| \leq n^{1 / p}\right) \\
& =n^{1-1 / p} \sum_{k=1}^{n} E|X| I\left(k-1<|X|^{p} \leq k\right)
\end{aligned}
$$

Since,

$$
\begin{aligned}
\sum_{k=1}^{\infty} k^{1-1 / p} E|X| I\left(k-1<|X|^{p} \leq k\right) & =\sum_{k=1}^{\infty} k^{1-1 / p} E|X|^{p}|X|^{1-p} I\left(k-1<|X|^{p} \leq k\right) \\
& \leq \sum_{k=1}^{\infty} k^{1-1 / p} E|X|^{p} I\left(k-1<|X|^{p} \leq k\right) k^{(1-p) / p} \\
& =\sum_{k=1}^{\infty} E|X|^{p} I\left(k-1<|X|^{p} \leq k\right) \\
& =E|X|^{p}<\infty .
\end{aligned}
$$

It follows from Kronecker lemma that

$$
n^{1-1 / p} \sum_{k=1}^{n} E|X| I\left(k-1<|X|^{p} \leq k\right) \rightarrow 0 \quad \text { as } n \rightarrow \infty .
$$

Hence, we get that

$$
n^{-1 / p} \max _{1 \leq j \leq n}\left|\sum_{i=1}^{j} E X_{i}^{(n)}\right| \rightarrow 0 \quad \text { as } n \rightarrow \infty .
$$

(2) when $1 \leq p<2$, by $E X_{n}=0$ and $E|X|^{p}<\infty$, then

$$
\begin{aligned}
n^{-1 / p} \max _{1 \leq j \leq n}\left|\sum_{i=1}^{j} E X_{i}^{(n)}\right| & \leq n^{-1 / p} \sum_{i=1}^{n}\left|E X_{i}^{(n)}\right| \\
& =n^{-1 / p} \sum_{i=1}^{n}\left|E\left(X_{i}-X_{i}^{(n)}\right)\right| \\
& =n^{1-1 / p} E\left(\left(X-n^{1 / p}\right) I\left(X>n^{1 / p}\right)+\left(X+n^{1 / p}\right) I\left(X<-n^{1 / p}\right)\right) \\
& \leq n^{1-1 / p} E\left(|X| I\left(|X|>n^{1 / p}\right)+n^{1 / p} I\left(|X|>n^{1 / p}\right)\right) \\
& \ll n^{1-1 / p} E|X|^{p} n^{(1-p) / p} I\left(|X|>n^{1 / p}\right) \\
& =E|X|^{p} I\left(|X|>n^{1 / p}\right) \rightarrow 0
\end{aligned}
$$

From (3.5) and (3.6), we easily know that (3.1) follows. 
By (3.1), for any $\varepsilon>0, n$ large enough, it follows from that

$$
\max _{1 \leq j \leq n}\left|\sum_{i=1}^{j} E X_{i}^{(n)}\right|<\frac{\varepsilon}{2} n^{1 / p}<\frac{\varepsilon}{2} n^{1 / p} \log n, \quad\left|\sum_{i=1}^{n} E X_{i}^{(n)}\right|<\frac{\varepsilon}{2} n^{1 / p}
$$

It follows from (3.1) that for $n$ large enough

$$
P\left(\max _{1 \leq j \leq n}\left|S_{j}\right|>\varepsilon n^{1 / p} \log n\right) \leq \sum_{j=1}^{n} P\left(\left|X_{j}\right|>n^{1 / p}\right)+P\left(\max _{1 \leq j \leq n}\left|S_{j}^{(n)}-E S_{j}^{(n)}\right|>\frac{\varepsilon}{2} n^{1 / p} \log n\right)
$$

Hence, we need only to prove that

$$
I \triangleq \sum_{n=1}^{\infty} n^{-1} \sum_{j=1}^{n} P\left(\left|X_{j}\right|>n^{1 / p}\right)<\infty .
$$

and

$$
\left.I I \triangleq \sum_{n=1}^{\infty} n^{-1} P\left(\max _{1 \leq j \leq n}\left|S_{j}^{(n)}-E S_{j}^{(n)}\right|>\frac{\varepsilon}{2} n^{1 / p} \log n\right)\right)<\infty .
$$

By $E|X|^{p}<\infty$, then

$$
I \triangleq \sum_{n=1}^{\infty} n^{-1} \sum_{j=1}^{n} P\left(\left|X_{j}\right|>n^{1 / p}\right)<\sum_{n=1}^{\infty} P\left(|X|>n^{1 / p}\right) \ll E|X|^{p}<\infty .
$$

Denote that $\tilde{X}_{i}^{(n)}=X_{i}^{(n)}-E X_{i}^{(n)}, \tilde{S}_{j}^{(n)}=\sum_{i=1}^{j} \tilde{X}_{i}^{(n)}, \alpha=\frac{1}{p}$, then, we know that $E \tilde{X}_{i}^{(n)}=0$. It follows from Lemma 3.2 and Markov inequality that

$$
\begin{aligned}
I I & \triangleq \sum_{n=1}^{\infty} n^{-1} P\left(\max _{1 \leq j \leq n}\left|S_{j}^{(n)}-E S_{j}^{(n)}\right|>\frac{\varepsilon}{2} n^{1 / p} \log n\right) \\
& \ll \sum_{n=1}^{\infty} n^{-1-2 \alpha} \log ^{-2} n E\left(\max _{1 \leq j \leq n}\left|\tilde{S}_{j}^{(n)}\right|^{2}\right) \\
& \leq c \sum_{n=1}^{\infty} n^{-1-2 \alpha} \log ^{-2} n \log ^{2} n \sum_{i=1}^{n} E\left(\tilde{X}_{i}^{(n)}\right)^{2} \\
& \leq c \sum_{n=1}^{\infty} n^{-2 \alpha} E|X|^{2} I\left(|X| \leq n^{1 / p}\right)+c \sum_{n=1}^{\infty} P\left(|X|>n^{1 / p}\right) \\
& \leq c \sum_{n=1}^{\infty} n^{-2 \alpha} \sum_{k=1}^{n} E|X|^{2} I\left(k-1<|X|^{p} \leq k\right)+c E|X|^{p} \\
& \leq c \sum_{k=1}^{\infty} E|X|^{2} I\left(k-1<|X|^{p} \leq k\right) \sum_{n=k}^{\infty} n^{-2 \alpha} \\
& \leq c \sum_{k=1}^{\infty} k^{-2 \alpha+1} E|X|^{2} I\left(k-1<|X|^{p} \leq k\right) \\
& =c \sum_{k=1}^{\infty} k^{-2 \alpha+1} E|X|^{p}|X|^{2-p} I\left(k-1<|X|^{p} \leq k\right) \\
& \leq c \sum_{k=1}^{\infty} k^{-2 \alpha+1} E|X|^{p} k^{(2-p) / p} I\left(k-1<|X|^{p} \leq k\right) \\
& =c \sum_{k=1}^{\infty} E|X|^{p} I\left(k-1<|X|^{p} \leq k\right) \\
& \ll c E|X|^{p}<\infty . \\
& =\infty(k)
\end{aligned}
$$


The proof of Theorem 2.1 is complete.

Remark 3.1 Theorem 2.1 shows that when $\alpha p=1$, Baum and Katz complete convergence theorem for pairwise NQD random variable sequences still holds true under the stronger condition. The result generalizes and extends the corresponding result of $\mathrm{Wu}$ [5] for sequences of pairwise NQD random variables with identically distributed.

Remark 3.2 Under the conditions of Theorem 2.1, it is well known that (2.1) holds for $0<p<1$ without the factor $\log n$, i.e.

$$
\sum_{n=1}^{\infty} n^{-1} P\left(\max _{1 \leq j \leq n}\left|S_{j}\right|>\varepsilon n^{1 / p}\right)<\infty \quad \text { for } 0<p<1 \text { and any } \varepsilon>0 .
$$

Proof of Theorem 2.2 For all $\varepsilon>0$, from (2.1), we obtain that

$$
\sum_{n=1}^{\infty} n^{-1} P\left(\max _{1 \leq j \leq n}\left|S_{j}\right|>\varepsilon n^{1 / p} \log n\right)<\infty .
$$

Then we know that

$$
\begin{aligned}
\infty & >\sum_{n=1}^{\infty} n^{-1} P\left(\max _{1 \leq j \leq n}\left|S_{j}\right|>\varepsilon n^{1 / p} \log n\right) \\
& =\sum_{i=0}^{\infty} \sum_{n=2^{i}}^{2^{i+1}-1}\left(2^{i+1}-1\right)^{-1} P\left(\max _{1 \leq j \leq n}\left|S_{j}\right|>\varepsilon n^{1 / p} \log n\right) \\
& \geq \frac{1}{2} \sum_{i=1}^{\infty} P\left(\max _{1 \leq j \leq 2^{i}}\left|S_{j}\right|>\varepsilon 2^{(i+1) / p} \log \left(2^{i+1}\right)\right) .
\end{aligned}
$$

It follows from Borel-Cantelli lemma that

$$
P\left(\max _{1 \leq j \leq 2^{i}}\left|S_{j}\right|>\varepsilon 2^{(i+1) / p} \log \left(2^{i+1}\right), \text { i.o. }\right)=0 .
$$

Hence,

$$
\lim _{i \rightarrow \infty} \frac{\max _{1 \leq j \leq 2^{i}}\left|S_{j}\right|}{2^{(i+1) / p} \log \left(2^{i+1}\right)}=0 . \quad \text { a.s. }
$$

For all positive integers $n$, there exists a non-negative integer $i_{0}$, such that $2^{i_{0}-1} \leq n<2^{i_{0}}$.

Thus

$$
\begin{aligned}
\max _{2^{i_{0}-1} \leq n \leq 2^{i_{0}}} \frac{\left|S_{n}\right|}{n^{1 / p} \log n} & \leq \frac{\max _{1 \leq j \leq 2^{i_{0}}}\left|S_{j}\right|}{2^{\left.i_{0}-1\right) / p} \log \left(2^{i_{0}-1}\right)} \\
& \leq 2^{\frac{2}{p}} \frac{\max _{1 \leq j \leq 2^{i_{0}}}\left|S_{j}\right|}{2^{\left(i_{0}+1\right) / p} \log \left(2^{i_{0}+1}\right)}\left(\frac{i_{0}+1}{i_{0}-1}\right) \\
& \rightarrow 0 . \text { a.s. }
\end{aligned}
$$

We have

$$
\lim _{n \rightarrow \infty} \frac{\left|S_{n}\right|}{n^{1 / p} \log n}=0 . \quad \text { a.s. }
$$

The proof of Theorem 2.2 is complete. 
Remark 3.3 Under the conditions of Theorem 2.2, it is well known that (2.2) holds for $0<p<1$ without the factor $\log n$, i.e.

$$
\lim _{n \rightarrow \infty} \frac{S_{n}}{n^{1 / p}}=0 . \quad \text { a.s. }
$$

Remark 3.4 Since NOD random variable sequences and NA random variable sequences are the most important and special cases of pairwise NQD random variable sequences, then we have the following results as two corollaries of Theorems 2.1 and 2.2.

Corollary 3.5 Let $\left\{X_{n} ; n \geq 1\right\}$ be a sequence of non-identically distributed NOD (or NA) random variables with $\left\{X_{n} ; n \geq 1\right\} \pi X$ and satisfying the conditions of Theorem 2.1, then

$$
\sum_{n=1}^{\infty} n^{-1} P\left(\max _{1 \leq j \leq n}\left|S_{j}\right|>\varepsilon n^{1 / p} \log n\right)<\infty \quad \text { for all } \quad \varepsilon>0 .
$$

Corollary 3.6 Let $\left\{X_{n} ; n \geq 1\right\}$ be a sequence of non-identically distributed NOD (or NA) random variables satisfying the conditions of Corollary 3.3, then,

$$
\lim _{n \rightarrow \infty} \frac{S_{n}}{n^{1 / p} \log n}=0 . \quad \text { a.s. }
$$

\section{Acknowledgements}

This study was supported by the National Natural Science Foundation of China (11061012), Project supported by Program to Sponsor Teams for Innovation in the Construction of Talent Highlands in Guangxi Institutions of Higher Learning, the Support Program of the New Century Guangxi China Ten-hundred-thousand Talents Project (2005214), and the Guangxi China Science Foundation (2010GXNSFA013120). The authors are very grateful to the referees and the editors for their valuable comments and some helpful suggestions that improved the clarity and readability of the article.

\section{Author details}

${ }^{1}$ School of Mathematics Science, University of Electronic Science and Technology of China, Chengdu 610054, PR China ${ }^{2}$ College of Science, Guilin University of Technology, Guilin 541004, PR China

\section{Authors' contributions}

$\mathrm{HH}$, DW and QW carried out the design of the study and performed the analysis. QZ participated in its design and coordination. All authors read and approved the final manuscript.

\section{Competing interests}

The authors declare that they have no competing interests.

Received: 4 May 2011 Accepted: 26 October 2011 Published: 26 October 2011

\section{References}

1. Lehmann, EL: Some concepts of dependence. Ann Math Stat. 43(3):1137-1153 (1966)

2. Joag Dev, K, Proschan, F: Negative association of random variables with applications. Ann Stat. 11(1), $286-295$ (1983). doi:10.1214/aos/1176346079

3. Matula, P: A note on the almost sure convergence of sums of negatively dependent random variables. Stat Prob Lett. 15(3), 209-213 (1992). doi:10.1016/0167-7152(92)90191-7

4. Wang, YB, Su, C, Liu, XG: On some limit properties for pairwise NQD sequences. Acta Math Appl Sinica. 21(3), 404-414 (1998)

5. Wu, QY: Convergence properties of pairwise NQD sequences. Acta Math Sinica. 45(3), 617-624 (2002)

6. Chen, PY: On the strong law of Large numbers for pairwise NQD random variables. Acta Math Sci. 25(3), 386-392 (2005)

7. Gan, SX, Chen, PY: Some limit theorems for sequences of pairwise NQD random variables. Acta Math Sci Ser B Engl Ed. 28(2), 269-281 (2008)

8. Hsu, PL, Robbins, H: Complete convergence and the law of large numbers. Proc Natl Acad Sci USA. 33(2), 25-31 (1947). doi:10.1073/pnas.33.2.25

9. Erdös, P: On a theorem of Hsu and Robbins. Ann Math Stat. 20, $286-291$ (1949). doi:10.1214/aoms/1177730037 
10. Baum, LE, Katz, M: Convergence rates in the law of large numbers. Trans Am Math Soc. 120(1), 108-123 (1965). doi:10.1090/S0002-9947-1965-0198524-1

doi:10.1186/1029-242X-2011-92

Cite this article as: Huang et al:: A note on the complete convergence for sequences of pairwise NQD random variables. Journal of Inequalities and Applications 2011 2011:92.

Submit your manuscript to a SpringerOpen ${ }^{\odot}$ journal and benefit from:

- Convenient online submission

- Rigorous peer review

- Immediate publication on acceptance

- Open access: articles freely available online

- High visibility within the field

- Retaining the copyright to your article

Submit your next manuscript at $\boldsymbol{\nabla}$ springeropen.com 